\title{
Spin Hall effect in a square 2DEG sample with a central defect
}

\author{
Son-Hsien Chen, Ivo Klik*, Ching-Ray Chang \\ Department of Physics, National Taiwan University, Taipei 106, Taiwan
}

\section{A R T I C L E I N F O}

Keywords:

Spin Hall effect

Rashba spin-orbit coupling

2DEG samples with defect

\begin{abstract}
A B S T R A C T
We study spin and charge accumulation and currents in two-dimensional electron gas (2DEG) with spin-orbit Rashba interactions. The Landauer-Keldysh formalism is employed to numerically compute the accumulations and currents in a 2DEG finite square sample with and without an embedded spinindependent hard-wall impurity. We find that under certain conditions the charge accumulation and the spin currents may be confined to a small region of the sample, between the defect and the positive lead. The conditions for the existence of this phenomenon are described.
\end{abstract}

(C) 2009 Published by Elsevier B.V.

\section{The model system}

The inversion symmetry of a two-dimensional electron gas (2DEG) leads to the Rashba spin-orbit (SO) coupling [1]. In this work, we present an interesting case of a steady state spin bond current confined by an impurity embedded in a small 2DEG sample. To this end we consider a finite spin-orbit interacting sample with an embedded central impurity. The sample is square, connected to two or four ideal leads, and the impurity is modeled by hard-wall boundary conditions. The system is thus arranged as a ballistic bridge consisting of the square 2DEG sample connected to the two or four leads in the customary Landauer setup. The size of the square sample is $(N-1)^{2} a^{2}$ where $a$ is the lattice constant and $N=8,9, \ldots, 30$ is the number of lattice nodes. The samples are either free of defects, or have a square defect of size $a^{2}$ if $N$ is even, and $4 a^{2}$ if $N$ is odd. In this manner, it is always possible to place the defect into the center of the sample. The width of the leads is $(N-1) a$, and the left and right leads carry the electric bias of $+e V_{p} / 2$ and $-e V_{p} / 2$, respectively. The top and bottom leads, if present, carry no bias.

The model sample is described by the Hamiltonian

$H=H_{R}+H_{\text {imp }}$,

where the first term describes the Rashba coupling potential and the second term the spin-independent impurity. A two-dimensional linear Rashba system in tight-binding approximation can be written as $[2,3]$

$H_{R}=\sum_{m \sigma} \varepsilon_{m} c_{m \sigma}^{+} c_{m \sigma}+\sum_{m m^{\prime} \sigma \sigma^{\prime}} c_{m \sigma}^{+} t_{m m^{\prime}}^{\sigma \sigma^{\prime}} c_{m^{\prime} \sigma^{\prime}}$

where $c_{m \sigma}{ }^{+}$and $c_{m \sigma}$ are creation and annihilation operators for $\sigma=+1$ (spin-up) and $\sigma=-1$ (spin-down) electrons, and $\varepsilon_{m}$ is the

\footnotetext{
* Corresponding author. Tel.: +8862 29995503; fax: +8862 29998764 .

E-mail address: iklik@phys.ntu.edu.tw (I. Klik).
}

on-site energy at site $m=\left(m_{x}, m_{y}\right)$. We choose $\varepsilon_{m}=0$ by selecting an overall energy shift $-4 t_{0}=4 \hbar / 2 m^{*} a^{2}$ where $m^{*}$ is the effective electron mass (see below). Let now $I_{S}$ be the identity matrix. Then the hopping terms of the Hamiltonian can be expressed in terms of the Rashba coupling strength $\alpha$ as

$t_{m m^{\prime}}^{\sigma \sigma^{\prime}}=\left\langle\sigma\left|-t_{0} I_{S}-i t_{S O}^{R} \sigma_{y}\right| \sigma^{\prime}\right\rangle$

for $m=m^{\prime}+e_{x}$, and

$t_{m m^{\prime}}^{\sigma \sigma^{\prime}}=\left\langle\sigma\left|-t_{0} I_{S}+i t_{S O}^{R} \sigma_{x}\right| \sigma^{\prime}\right\rangle$

for $m=m^{\prime}+e_{y}$. In these expressions $t_{0}=\hbar / 2 m^{*} a^{2}$ and $t_{S O}{ }^{R}=\alpha / 2 a$.

The impurity potential in the tight-binding approximation only contributes to the on-site energy, and can therefore be written as

$H_{i m p}=\sum_{m \sigma} V_{m} c_{m \sigma}^{+} c_{m \sigma}$

where $V_{m}=0$ if $m \neq m_{i m p}$ and $V_{m}=\infty$ if $m=m_{\text {imp. }}$.

Given the Hamiltonian we now employ the Landauer-Keldysh formalism [4,5] to obtain the spatial distribution of the spin accumulation and the spin bond current, so that the spin $S_{z}$ current, for example, is given by the expression

$J_{m m^{\prime}}^{S_{z}}=\frac{t_{0}}{4 \pi} \int_{E_{F}-e V / 2}^{E_{F}+e V / 2} d E \operatorname{Tr}_{s}\left\{\sigma_{z}\left[G_{m^{\prime} m}^{<}(E)-G_{m m^{\prime}}^{<}(E)\right]\right\}$

where the trace is taken over the spin $-\frac{1}{2}$ space, $G^{<}(E)$ is the lesser Green's function, and $\sigma_{z}$ is the Pauli matrix. The leads are assumed to be in thermal equilibrium so that, in lead $p$, where the positive bias $+e V_{p}$ is applied, the electrons are distributed according to the Fermi-Dirac distribution

$f_{p}(E)=1 /\left\{1+\exp \left[-\left(e-e V_{p}\right) / k_{B} T\right]\right\}$

The lesser Green's function is obtained from the kinetic equation

$G^{<}(E)=G^{r}(E) \sum^{<}(E) G^{r+}(E)$. 
The leads are taken into account by the self-energy [6]

$\Sigma(E)=\sum_{p} \sum_{p}(E)=\sum_{p} t_{0}^{2} g_{p}^{r}(E)$

both in the retarded Green's function

$G^{r}(E)=1 /[E-H-\Sigma(E)]$

and in the lesser self-energy

$\Sigma^{<}(E)=-\sum_{p}\left[\Sigma_{p}\left(E^{\prime}\right)-\Sigma_{p}^{+}\left(E^{\prime}\right)\right] \times f\left(E^{\prime}\right)$

where $E^{\prime}=E-e V_{p}$. The steady state spin accumulation and bond currents then follow from here by means of a numerical calculation.

The Landauer-Keldysh Green's function formalism yields the spin accumulation and the steady state spin bond current in the sample with and without an impurity. Our calculation is preformed with the conventional parameters for a two-dimensional gas confined in an InGaAs/InAlAs semiconductor heterostructure [7] grown along the [001] direction: the electron effective mass $m^{*}=0.05 m_{e}$ ( $m_{e}$ is the electron rest mass), and the lattice constant $a=3 \mathrm{~nm}$ which yields the hopping energy $t_{0}=84.68 \mathrm{meV}$. We set the Fermi wavelength [7] as $\lambda_{F}=14 a$ which corresponds to the Fermi energy $E_{F}=-3.8 t_{0}$. This is close to the band bottom at $E_{b}=-4 t_{0}$, so that the long wavelength approximation [5,7] is valid. The Rashba hopping strength $t^{R}=0.1 t_{0}$ yields the precession length $L_{S O}=15.7 a$. For purposes of comparison we have carried out our calculations with the highand low-bias values of $e V=0.4 t_{0}$ and $e V=10^{-3} t_{0}$, but only the low-bias data are discussed here in detail.

The symmetry properties of the Hamiltonian yield the symmetry properties of our numerical solutions: let therefore $\xi_{x}$ be a horizontal axis which passes through the center of the ballistic bridge and possibly through the center of the impurity as well. Then the local charge accumulation and the corresponding local non-equilibrium bond charge current are symmetric with respect to the current axis $\xi_{x}$, and so are the accumulation and bond spin current of the $y$ spin-component. The accumulations and bond spin currents of the $x$ - and $z$-spin components, on the other hand, are antisymmetric with respect to $\xi_{x}$.

\section{Numerical results}

In this section, we present the results of our numerical calculations. Unless otherwise specified we consider here the two leads (left and right) and low bias, small sample arrangement. We concentrate on small samples so that the effect of the defect is not distorted by the precessional and wave interference effects so characteristic of larger samples.

We first discuss the charge accumulation and charge current in a sample with and without a central defect. These two quantities are interesting in that their behavior is diametrically opposed to the behavior of the spin accumulation and spin current. In the absence of the defect the charge accumulation forms, as expected, a band pattern with charge depleted regions along the upper and lower edges of the sample. The highest charge accumulation is in the central region of the sample, along the axis $\xi_{x}$. The charge current flows from left to right, with maximum current long the $\xi_{x}$ axis, and zero current along the upper and lower edges of the sample. This current pattern is only slightly perturbed by the presence of the defect, since the current simply flows around it. However, the charge accumulation in this case is confined entirely to the left of the defect, and the rest of the sample is fully depleted of charge (see Fig. 1). We shall show presently that in the case of spin this confinement phenomenon is observed in the steadystate bond current, and not in the accumulation pattern as is the case with the electric charge.

Let us consider first the accumulation of the $z$-spin component: in the absence of a defect we observe a two band pattern, with the upper band composed of negatively oriented spins and the lower band of positively oriented spins. The highest spin concentration

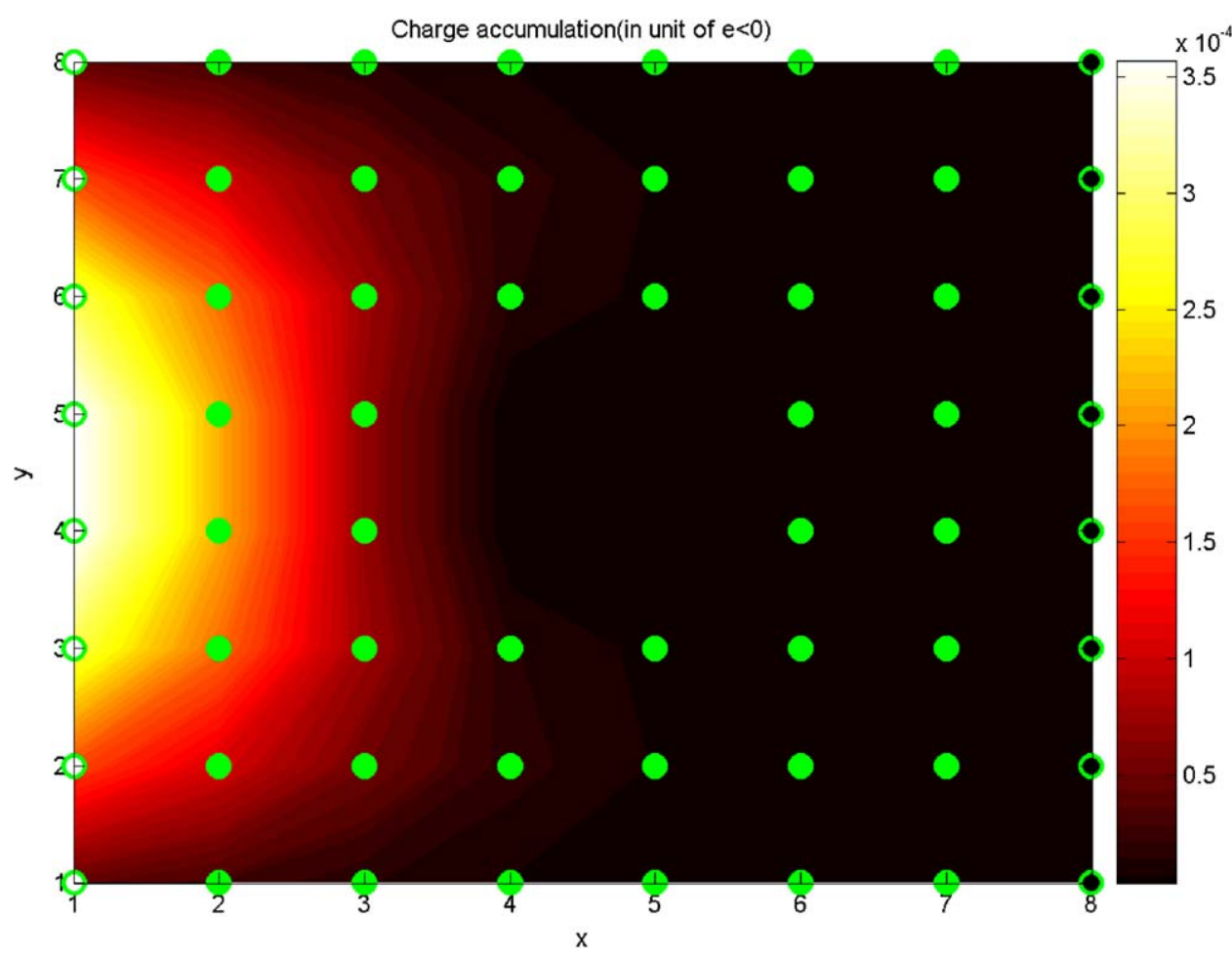

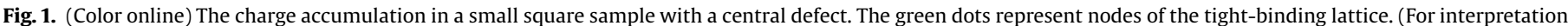
of the references to color in this figure legend, the reader is referred to the web version of this article). 


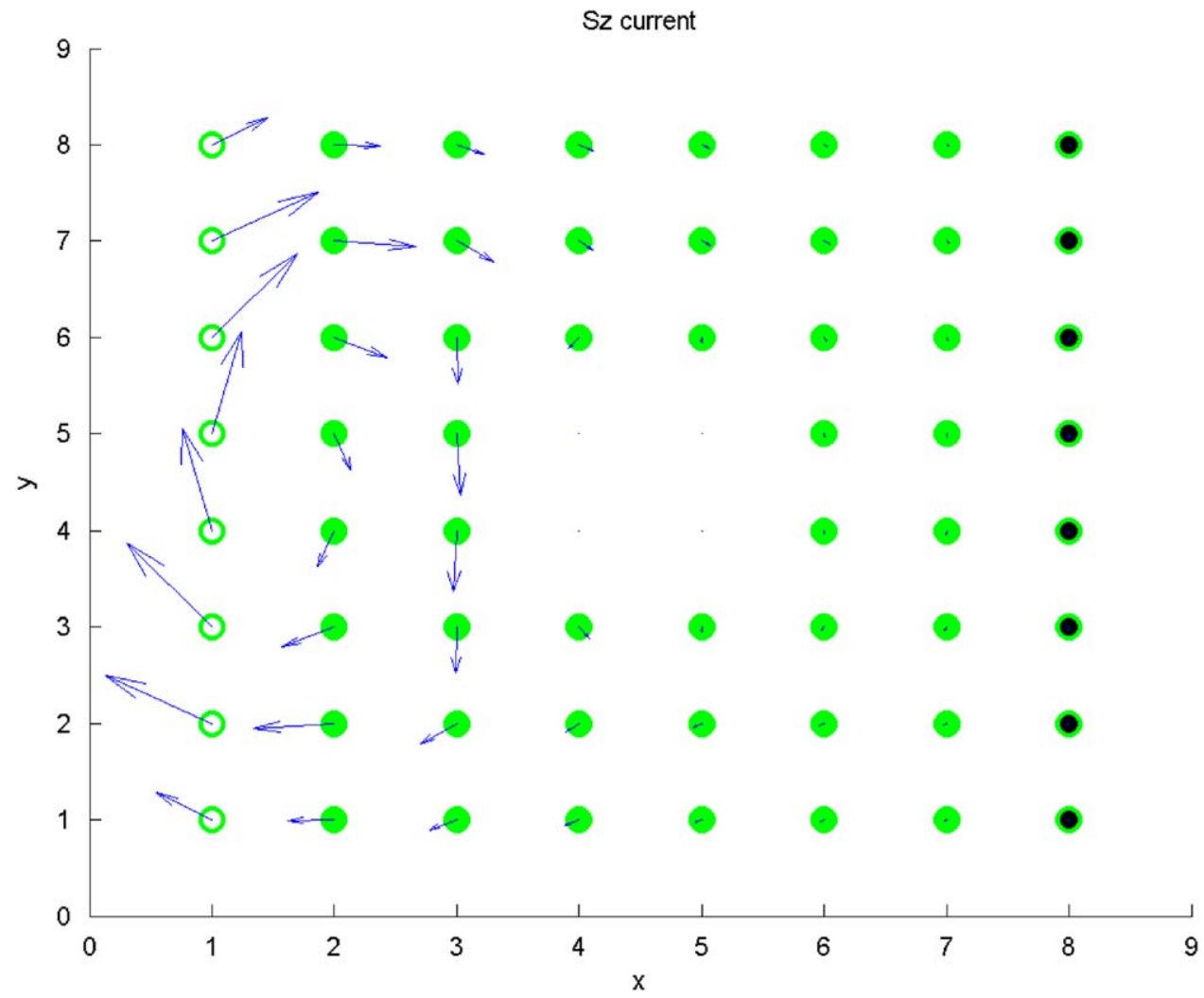

Fig. 2. (Color online) The bond current of the $z$-spin component in a small square sample with a central defect. The white and black dots in the leftmost and rightmost node columns are an artifact of the plotting software, and have no physical significance.

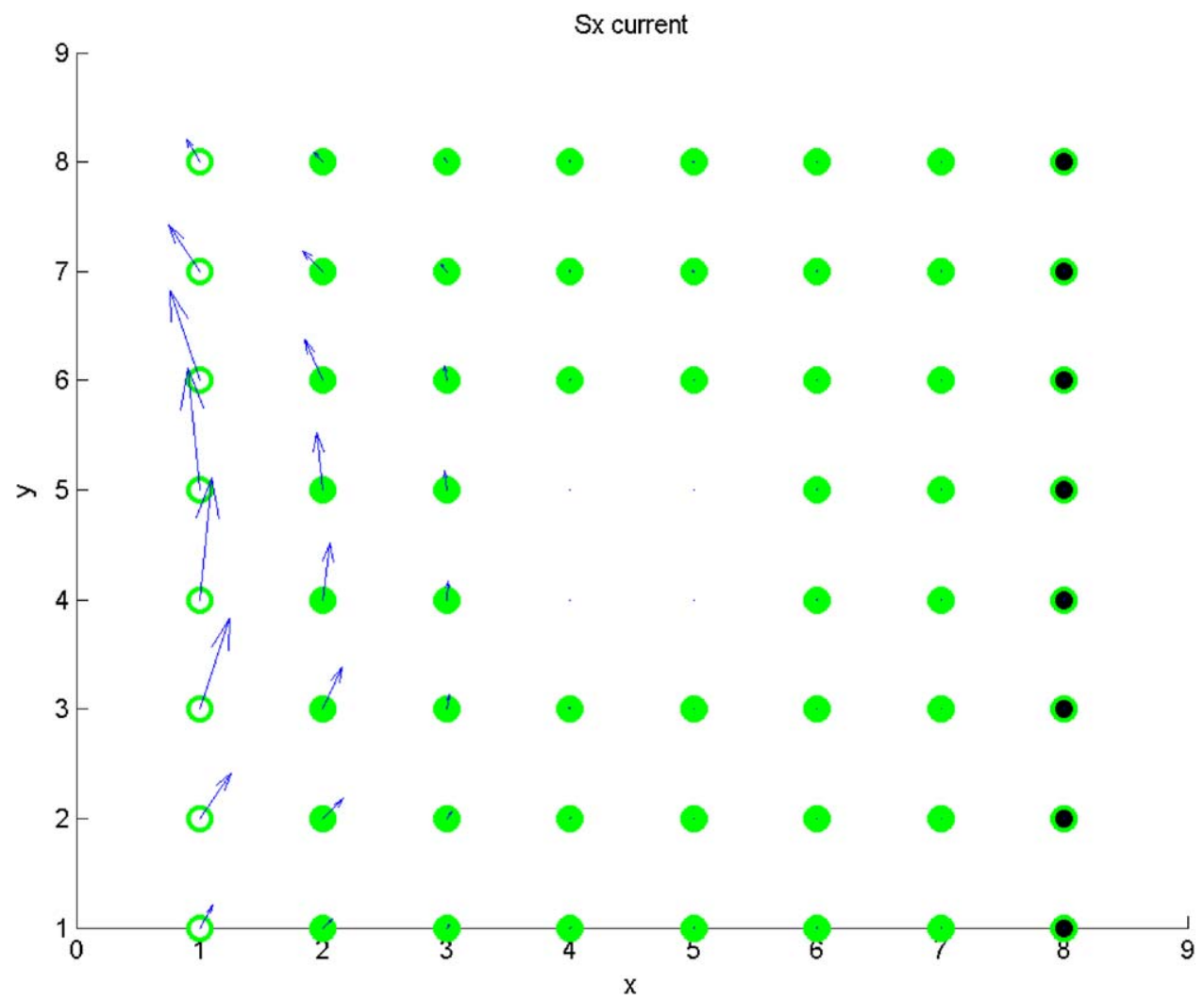

Fig. 3. Same as Fig. 2, but for the $x$-spin component. 


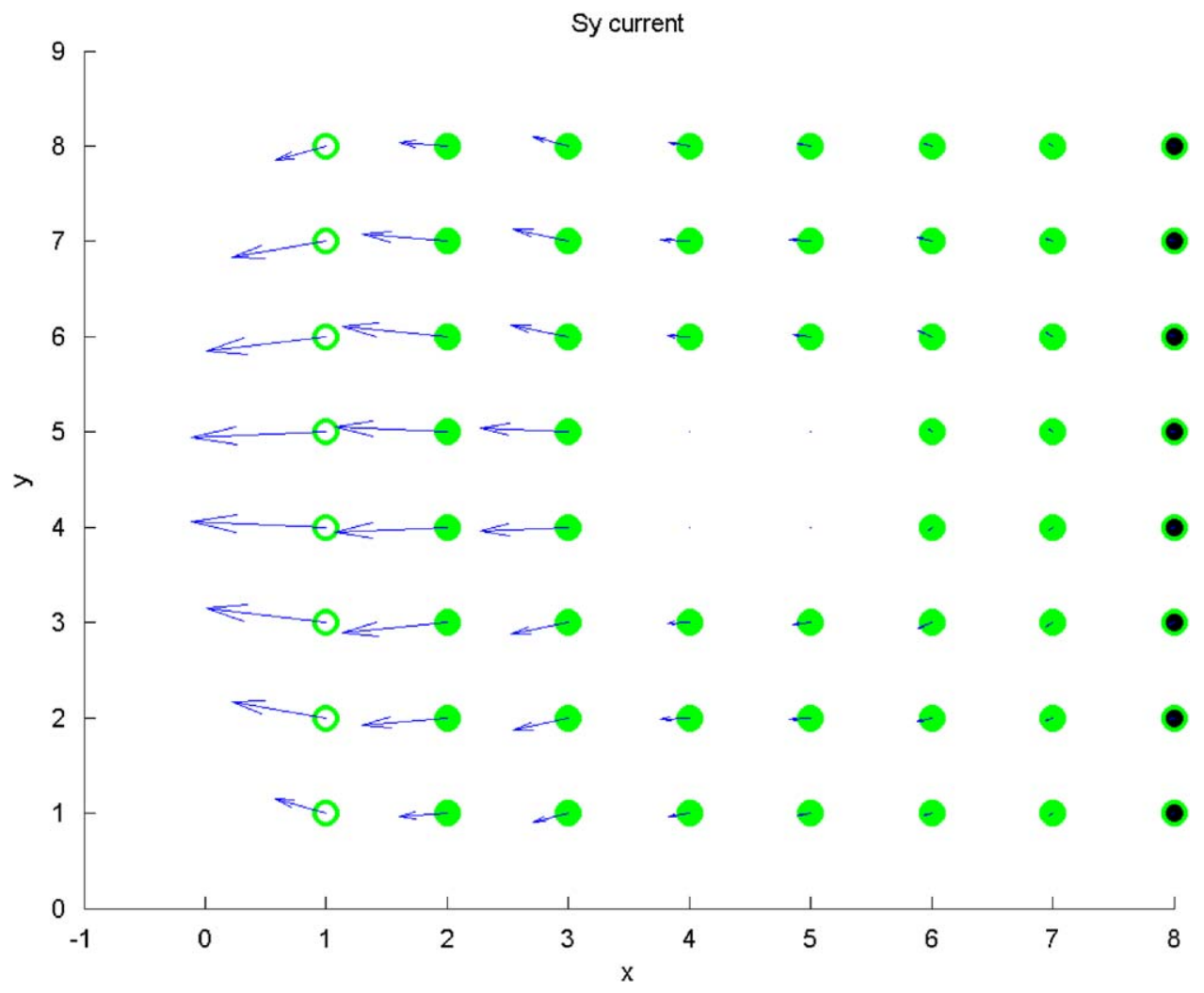

Fig. 4. Same as Fig. 2, but for the $y$-spin component.

is along the centerline of each band. A defect, if present, induces a dipole [8] which is oriented inversely to the orientation of the sample, so that it decreases the spin density in both bands. Indeed, in some cases the induced dipole is so strong that it reverses the orientation of the entire sample. The current of the $z$-spin component is antisymmetric with respect to the axis $\xi_{x}$, and forms a single vertex structure within the sample. The effect of the defect is apparent from Fig. 2 which shows the spin bond current to be entirely confined to the region left of the defect. Its symmetry properties are not affected, so that the current still forms a single vertex. With increasing sample size, the current gradually leaks to the right of the sample where it forms a twovertex pattern.

The $x$-spin component enjoys the same antisymmetric properties as the $z$-component. The unperturbed accumulation pattern is again comprised of two horizontal bands, with each band being formed by spin-up (upper left and lower right sample corners) and spin-down regions. The defect induces a quadrupole which entirely reverses the sample, with spin-up regions becoming spindown regions and vice versa. No confinement takes place. The unperturbed bond spin current is confined to the vertical edges of the sample, where it runs from bottom to top. The inclusion of the defect (see Fig. 3) leaves the left current pattern essentially unchanged, but fully suppresses the current pattern along the right edge of the sample. The spin current again leaks to the right of the defect in sufficiently large samples.

The $y$-spin component precesses parallel to the Rashba field, and its dynamics are therefore determined purely by the boundary conditions. We also recall that the accumulation and bond spin currents are here symmetric with respect to the current axis $\xi_{x}$. The unperturbed accumulation pattern is composed of two horizontal spin-up stripes separated by a horizontal spin-down region. The inclusion of the defect has scant effect on this accumulation pattern. The bond spin current is in this case very similar to the charge current described above, but with opposite orientation. According to Fig. 4, this current is again confined to the left of the sample defect, but in large samples a two-vertex structure forms around the imperfection.

\section{Summary}

In this work we have brought together four cases of spatial confinement of a physical quantity due to the presence of a sample defect. We have shown here that both charge accumulation and spin bond current may be confined in a small sample to a space delimited by the positively charged lead and the central defect. No clear physical explanation of this phenomenon is as yet known to us, though it appears certain that it is determined by the boundary conditions. In this work, we have discussed only low bias, two-leads samples, but the confinement exists also in highbias samples. It vanishes in large samples with small defects, preferentially at increased bias, and also in samples with four leads which were described in the introduction.

\section{References}

[1] E.I. Rashba, Sov. Phys. Solid State 2 (1960) 1109.

[2] S.H. Chen, M.H. Liu, K.W. Chen, C.R. Chang, J. Appl. Phys. 103 (2008) 07 B721.

[3] S.H. Chen, M.H. Liu, C.R. Chang, Phys. Rev. B 76 (2007) 075322.

[4] B.K. Nikolic, S. Souma, P. Zarbo, J. Sinova, Phys. Rev. Lett. 95 (2005) 046601.

[5] B.K. Nikolic, P. Zarbo, S. Souma, Phys. Rev. B 73 (2006) 075303.

[6] S. Datta, Electronic Transport in Mesoscopic Systems, Cambridge University Press, Cambridge, 1995.

[7] J. Nitta, T. Akazaki, H. Takayanagi, T. Enoki, Phys. Rev. Lett. 78 (1997) 1335.

[8] A.G. Mel'shukov, L.Y. Wang, C.S. Chu, Phys. Rev. B 75 (2007) 085315. 